\title{
Caracterización clínica de los pacientes con diagnóstico de leucemia mieloide crónica tratados en el Instituto Nacional de Cancerología
}

- Humberto Martínez Cordero, Leonardo Enciso Olivera, Jennifer Arenas, Carlos Camargo, Antonio Huertas

Correo electrónico: humbertomartinez48@hotmail.com

Introducción: la leucemia mieloide crónica (LMC) es una enfermedad que tratada de forma adecuada puede mostrar un comportamiento benigno e indolente en la mayoría de los pacientes. El objetivo de este estudio es caracterizar a la población con LMC tratada en el Instituto Nacional de Cancerología de Colombia hasta diciembre de 2016.

Métodos: estudio de corte transversal conducido en el Instituto Nacional de Cancerología con pacientes atendidos en la consulta externa y en hospitalización del servicio de hematología.

Resultados: un total de 182 pacientes fueron identificados, de los cuales 80 fueron mujeres. La mediana de edad al diagnóstico fue de 54 años. El 97,3\% de los pacientes se encontraban en fase crónica y el $2,7 \%$, en fase avanzada. Solo un $30,02 \%$ se hallaron en la primera línea de tratamiento. De los pacientes con enfermedad cuyo tratamiento es superior a 18 meses, tan solo un $62,50 \%$ estaba en respuesta citogenética completa o mejor. El 38,5\% tuvo una respuesta molecular mayor y el $18,1 \%$ mostraba enfermedad molecularmente indetectable. El 25,3\% solo había alcanzado respuesta hematológica y el 7,7\%, ningún tipo de respuesta. Un 35,7\% había tenido pérdida de la respuesta desde el último control. En cuanto al tratamiento, el 37,9\% recibía dasatinib; el 32,54\%, nilotinib; el 23,6\%, imatinib; y dos pacientes estaban en período postrasplante. La mayor razón para cambio del tratamiento fue el fallo en un $85,4 \%$, intolerancia en un $14,6 \%$, y la más frecuente fue toxicidad hematológica en un $55 \%$, seguida de edema en un $11 \%$ y de toxicidad renal en un $6 \%$. Se tuvo acceso a evaluar la adherencia en el $76 \%$ de los pacientes. Por la escala de Morisky-Green Levine, fue del 83,5\% y, por SMAQ, que es más estricta, del $72,7 \%$. El $61,9 \%$ de los pacientes tuvieron problemas con la entrega del medicamento por parte de su compañía aseguradora.

Conclusión: los desenlaces en el tratamiento de la LMC son diferentes en nuestra población en comparación con estudios de referencia internacionales. El conocimiento de nuestros datos basales ha generado la iniciativa del programa de LMC que hoy funciona en el Instituto Nacional de Cancerología.

\section{Aplasia severa de médula ósea (ASMO) en Colombia: características y tratamiento en seis centros}

\section{- Virginia Abello, Carlos Pardo, Kenny Mauricio Gálvez, Mónica Osuna, Paola Omaña, Claudia Sossa}

Correo electrónico: virginia.abello@gmail.com

\begin{abstract}
Introducción: la ASMO es una enfermedad hematológica de baja frecuencia asociada con morbimortalidad significativa. La destrucción por mecanismos inmunológicos de las células progenitoras hematopoyéticas parece jugar un papel fundamental en la fisiopatología de la enfermedad, por lo tanto, el tratamiento inmunosupresor es uno de los pilares del manejo. En Colombia, no existe un registro nacional que nos permita conocer las características de estos pacientes. El objetivo de este estudio fue analizar la experiencia en el manejo de este tipo de enfermos en varios centros del país, como un piloto inicial de un posible registro nacional de ASMO.
\end{abstract}

Métodos: se trata de un estudio observacional retrospectivo, efectuado en seis instituciones de tres ciudades del país. Se recolectó información de pacientes atendidos en estas de 2013 a 2018.
Resultados: se analizó la información de 28 pacientes en seis centros, la edad media fue 25 años (rango 6-68 años), 18 eran mujeres. El tiempo del inicio de los síntomas al diagnóstico fue 83 días (rango 7-567). Los síntomas más importantes fueron sangrado e infecciones. En 6 pacientes se encontraron clones de HPN. Veintiún pacientes recibieron en primera línea globulina antilinfocítica ( 9 de conejo, 10 de caballo, 2 combinada con eltrombopag); 2 , ciclosporina y prednisona; 2 , trasplante alogénico; y 2 , otras terapias. Diez pacientes tuvieron respuesta completa; 8 , parcial; y 10 no respondieron. En el último control, 20 estaban vivos, 5 habían muerto. Conclusión: esta información preliminar muestra que hay una demora significativa en el diagnóstico e inicio de tratamiento en pacientes con ASMO, lo cual podría explicar las tasas de respuesta aparentemente inferiores a lo esperado para la literatura. 